\title{
Association between serum bone biomarker levels and ultrasonographic response in abatacept-treated rheumatoid arthritis patients
}

Shin-Ya Kawashiri ( $\nabla$ shin-ya@hotmail.co.jp )

Nagasaki University Graduate School of Biomedical Sciences https://orcid.org/0000-0002-2606-995X

\section{Yushiro Endo}

Nagasaki University Graduate School of Medical Sciences

Ayako Nishino

Nagasaki University Graduate School of Medical Sciences

Momoko Okamoto

Nagasaki University Graduate School of Medical Sciences

\section{Sosuke Tsuji}

Nagasaki University Graduate School of Medical Sciences

\section{Ayuko Takatani}

Nagasaki University Graduate School of Medical Sciences

\section{Toshimasa Shimizu}

Nagasaki University Graduate School of Medical Sciences

\section{Remi Sumiyoshi}

Nagasaki University Graduate School of Medical Sciences

\section{Tomohiro Koga}

Nagasaki University Graduate School of Medical Sciences

Naoki Iwamoto

Nagasaki University Graduate School of Medical Sciences

Kunihiro Ichinose

Nagasaki University Graduate School of Medical Sciences

Mami Tamai

Nagasaki University Graduate School of Medical Sciences

\section{Hideki Nakamura}

Nagasaki University Graduate School of Medical Sciences

\section{Tomoki Origuchi}

Nagasaki University Graduate School of Medical Sciences

\section{Toshiyuki Aramaki}

Kyushu Multicenter Rheumatoid Arthritis Ultrasound Prospective Observational Cohort Study Group 
Kyushu Multicenter Rheumatoid Arthritis Ultrasound Prospective Observational Cohort Study Group

\section{Tamami Yoshitama}

Kyushu Multicenter Rheumatoid Arthritis Ultrasound Prospective Observational Cohort Study Group

\section{Nobutaka Eiraku}

Kyushu Multicenter Rheumatoid Arthritis Ultrasound Prospective Observational Cohort Study Group

Naoki Matsuoka

Kyushu Multicenter Rheumatoid Arthritis Ultrasound Prospective Observational Cohort Study Group

Akitomo Okada

Kyushu Multicenter Rheumatoid Arthritis Ultrasound Prospective Observational Cohort Study Group

\section{Keita Fujikawa}

Kyushu Multicenter Rheumatoid Arthritis Ultrasound Prospective Observational Cohort Study Group Hiroaki Hamada

Kyushu Multicenter Rheumatoid Arthritis Ultrasound Prospective Observational Cohort Study Group

\section{Shuji Nagano}

Kyushu Multicenter Rheumatoid Arthritis Ultrasound Prospective Observational Cohort Study Group Yoshifumi Tada

Kyushu Multicenter Rheumatoid Arthritis Ultrasound Prospective Observational Cohort Study Group

\section{Takahiro Maeda}

Nagasaki University Graduate School of Medical Sciences

\section{Atsushi Kawakami}

Nagasaki University Graduate School of Medical Sciences

\section{Research article}

Keywords: rheumatoid arthritis, musculoskeletal ultrasound, abatacept, DKK-1, osteoprotegerin, sclerostin

Posted Date: May 1st, 2020

DOl: https://doi.org/10.21203/rs.3.rs-25419/v1

License: (c) (i) This work is licensed under a Creative Commons Attribution 4.0 International License. Read Full License 


\section{Abstract \\ Background}

To evaluate the effect of treatment on bone biomarkers and explore whether bone biomarkers are associated with therapeutic response in rheumatoid arthritis (RA) patients treated with abatacept.

\section{Methods}

We enrolled 59 RA patients treated with abatacept from a multicenter prospective ultrasound cohort study of patients who received biologic or targeted synthetic disease-modifying antirheumatic drug (DMARD) therapy. We evaluated the patients' clinical disease activity and musculoskeletal ultrasound (MSUS) scores. The serum concentrations of five bone biomarkers were evaluated (dickkopf-1 [Dkk-1], sclerostin [SOST], osteocalcin [OC], osteopontin [OPN], and osteoprotegerin [OPG]) by multiplex bead assays at baseline, 3 , and 6 months: the change over 6 months was defined as the $\Delta$ value. 'Power Doppler (PD) responder' was defined as a patient whose $\Delta$ total PD score over 6 months was greater than the median change.

\section{Results}

Abatacept significantly improved clinical disease activity as well as the MSUS score over 6 months. Serum OPG was significantly elevated at 6 months after the introduction of abatacept $(p=0.016)$. The $\triangle$ SOST and $\triangle O P G$ values were negatively correlated with the $\Delta$ total PD score ( $r s=-0.31, p<0.05$ and $r s$ $=-0.34, p<0.01$, respectively). The serum Dkk-1 at baseline was significantly lower in the PD responders $(n=30)$ compared to the non-PD responders $(n=29)(p=0.026)$. A multivariate logistic regression analysis showed that the serum Dkk-1 at baseline (odds ratio 0.50, $p=0.043$ ) was an independent predictor of PD responder status.

\section{Conclusion}

Serum levels of bone biomarkers may be useful for predicting RA patients' ultrasonographic response to abatacept.

\section{Background}

Rheumatoid arthritis (RA) is characterized by persistent synovitis, systemic inflammation, and autoantibodies [1]. Uncontrolled active RA causes joint damage, disability, decreased quality of life, and comorbidities. The tight control of the disease activity of RA following the treat-to-target (T2T) strategy is thus recommended [2]. Advances in the treatment of RA, such as biological disease-modifying antirheumatic drugs (bDMARDs), have provided better clinical outcomes, including the achievement of 
clinical remission, the prevention of joint damage, and the preservation of function for individuals with RA.

Abatacept, a fusion protein of cytotoxic T lymphocyte-associated antigen-4 (CTLA-4) and immunoglobulin $\mathrm{G} 1$, selectively modulates the CD80/CD86:CD28 costimulatory signal required for full Tcell activation [3]. Abatacept is an effective treatment for patients with RA, according to both clinical trials [4-7] and practice [8]. Abatacept is thus recommended as one of the first-line bDMARDs for RA [9]. Several studies showed that abatacept strongly inhibits radiographic progression in patients with RA [58]. CTLA-4 is suggested to be an anti-osteoclastogenic molecule that directly binds osteoclast precursor cells and inhibits their differentiation $[10,11]$.

Bone damage in RA is characterized by articular erosions, periarticular bone loss, and secondary osteoporosis caused by chronic inflammation [12]. In the bone metabolism of RA osteoclastogenesis/bone resorption is promoted and bone formation is inhibited. Among the underlying mechanisms, important roles are played by the interaction of immune responses mediated by proinflammatory cytokines such as tumor necrosis factor (TNF), interleukin (IL)- 6 and IL-17, and bone biomarkers such as receptor activator of nuclear factor kappa-B ligand (RANKL), osteoprotegerin (OPG), dickkopf-1 (Dkk-1), and sclerostin (SOST) [12-15]. Effects of bDMARDs including TNF inhibitors [16, 17] and IL-6 inhibitors [18-20] on bone biomarkers in patients with RA have been reported, but the effect of abatacept on bone biomarkers in patients with RA has not been investigated. Here, we measured the serum levels of five bone biomarkers (Dkk-1, SOST, osteocalcin [OC], osteopontin [OPN], and OPG) using a multiplex bead assay (bone panel) in RA patients treated with abatacept.

Dkk1 and SOST inhibit the Wnt signaling pathway regulating bone formation [12, 14, 15]. OC is an important component of bone matrix protein, synthesized mainly in osteoblasts [12, 21], and it is a biomarker of bone formation. OPG is a soluble decoy RANKL receptor that inhibits RANKL function, and it is highly involved in inflammatory bone resorption by interfering with RANK-RANKL binding $[12,14,15]$. OPN is strongly expressed in bone, where it promotes the adhesion of osteoclasts to the mineralized matrix regulating bone resorption and formation [22]. OPN has also been recognized as an important proinflammatory mediator which participates in pathological processes such as inflammatory reactions, vasospasm formation, and bone damage [23, 24].

To achieve the goal of the T2T strategy, the adequate management of disease activity requires a sensitive and accurate assessment of arthritis. Imaging plays an important role in this assessment. Musculoskeletal ultrasound (MSUS) has been widely applied in clinical settings as an imaging modality for patients with rheumatic diseases $[25,26]$. Compared to clinical and radiographic examinations, MSUS provides a straightforward and more accurate detection of both inflammation and damage at the joint level $[25,26]$. We have conducted a multicenter prospective observational cohort study of patients with active RA who received bDMARDs or targeted synthetic DMARDs (tsDMARDs) therapy at 27 participating rheumatology centers from the Kyushu region of Japan since June 2013 [27, 28]. We evaluated the therapeutic efficacy of the bDMARDs and tsDMARDs by clinical measurements, MSUS, and biomarker 
assessments. A multicenter collaborative study that prospectively evaluates disease activity by using MSUS standardized at a high level is rare, even worldwide.

In the present study, we evaluated the effect of abatacept treatment on bone biomarkers and explored whether bone biomarkers are associated with the therapeutic response in RA patients treated with abatacept.

\section{Patients And Methods}

\section{Patients}

This study is part of an ongoing non-randomized multicenter prospective cohort study of patients with active RA who received bDMARD or tsDMARD therapy at 27 participating rheumatology centers from the Kyushu region of Japan since June 2013 [27, 28]. We evaluated therapeutic efficacy by determining the patients' clinical disease activity, MSUS score, and serum biomarkers at baseline and at 3, 6, 9, 12, 18, and 24 months starting from the initiation of new bDMARD or tsDMARD. For the present study, we enrolled the 59 consecutive Japanese patients with active RA who were treated with abatacept and had continued the treatment for $>6$ months at 10 participating rheumatology centers during the period from June 2013 to March 2016. All patients were required to satisfy the 1987 American College of Rheumatology and/or 2010 American College of Rheumatology (ACR)/EULAR criteria for RA [29]. Abatacept was administered as recommended by the manufacturers: $125 \mathrm{mg}$ via subcutaneous injection weekly or 500-750 mg via intravenous infusion every 4 weeks. We excluded patients who were treated with an oral bisphosphonate during the study period or treated with intravenous bisphosphonates, antireceptor activator of nuclear factor kappa-B ligand (RANKL) antibodies, or parathyroid hormone (PTH) agents.

The study is registered with the University Hospital Medical Information Network Clinical Trials Registry (http://www.umin.ac.jp/ctr/, \#UMIN 000012524) and was approved by the Institutional Review Board of Nagasaki University (approval no. 13102866). All patients gave their signed informed consent to participate in accordance with the Helsinki Declaration.

\section{Clinical and laboratory assessments}

Disease activity was evaluated by each of the attending physicians (Japan College of Rheumatology [JCR]-certified rheumatologists) according to the Disease Activity Score (DAS) 28- C-reactive protein (CRP) value and Simple Disease Activity Index (SDAI) value at baseline and every 3 months after the introduction of abatacept. The treating physicians were different from the MSUS evaluators. The baseline MSUS scores were evaluated after the decision regarding the introduction of b/tsDMARDs therapy.

\section{Musculoskeletal ultrasound assessment}

The MSUS examination of each patient was performed by JCR-certified sonographers. At all of the participating institutions, a trained MSUS expert examined the patient in a situation recommended by the 
JCR guidelines, paying attention to factors that can affect PD results, including the room temperature, the last use of a nonsteroidal anti-inflammatory drug (NSAID), and hand position. Medium-level to high-level ultrasound machines were used (Toshiba AplioXG and Aplio300, GE Logic series 7 and 8 or Hitachi Ascendus, Avius, Noblus, and Hi Vision Preirus) with high-frequency (12-18.5 MHz) linear transducers. The Doppler parameters were adjusted according to the device used (range of pulse repetition frequency $500-1000 \mathrm{~Hz}$; Doppler frequency 6.1-10.0 MHz). There was no change in MSUS settings during the study.

Twenty-two joints including the metacarpophalangeal (MCP), proximal interphalangeal (PIP), and wrist joints of the bilateral hands were assessed by MSUS at baseline and at 3 and 6 months of treatment. The 22 joints were scanned on the dorsal aspect. Standardized joint and probe positions were used, based on a guideline published by the JCR. Each grayscale (GS) synovial hypertrophy and PD signal was scored semi-quantitatively on a scale from 0 to 3 [30]. The sum of the GS or PD scores was used as the indicator of US disease activity, described as the total GS score or total PD score. The total scores ranged from 0 to 66. We defined a PD responder at 6 months as a patient whose change in total PD score ( $\triangle$ total PD score) over 6 months was greater than the median change (i.e., a $\Delta$ total PD score less than or equal to 4 ) in all patients. We defined PD remission as a total PD score of 0 at 6 months. Interobserver reliability was confirmed in a previous investigation [27].

\section{Bone biomarker measurements}

We measured the concentrations of the following biomarkers by using serum stored on the same day as the patient's clinical evaluation. Rheumatoid factor (RF) was measured by the latex agglutination turbidimetric immunoassay (LATIA) (LZ test 'Eiken' RF). Anti-cyclic citrullinated peptide antibody (ACPA) was measured by a chemiluminescent immunoassay (CLEIA) (STACIA MEBLux test CCP). We performed the multiplex bead assays using diluted serum supernatants and a Milliplex MAP Human Bone Panel analyzed with a Bio-Plex® MAGPIX ${ }^{\circledR M}$ Multiplex Reader (Bio-Rad, Hercules, CA) according to the manufacturer's instructions. The bone biomarkers that could be measured by the bead panel included Dkk-1, SOST, OC, OPN, and OPG using a multi-suspension array. These bone biomarkers were also measured in 18 healthy age- and gender-matched volunteers (median age 70 years, 67\% females). The volunteers were recruited at medical check-ups in the town of Saza, Japan. The protocol was approved by the Nagasaki University Ethics Committee for Humans Subjects (approval no. 14051404).

\section{Statistical analyses}

Statistical analyses were performed using JMP Pro statistical software, ver. 11.0 (SAS, Cary, NC). Quantitative variables are presented as medians and interquartile ranges (IQRs). Categorical variables are presented as percentages. We used the Mann-Whitney U-test for comparisons between independent medians, and we used the Chi-square test for the evaluation of the associations between categorical variables. Correlations were assessed with Spearman's correlation coefficient. The changes in clinical disease activity indices, MSUS scores, or serum concentrations of bone biomarkers over 6 months were analyzed using the Wilcoxon signed ranks test. We attempted to identify any variables that were 
independently predictive of the PD responder status at 6 months from the patients' baseline characteristics by performing a multivariate logistic regression analysis. All variables with a $p$-value $<0.1$ in a univariate analysis were used in the multivariate models, but the total GS score was excluded as a confounding factor of the total PD score. P-values $<0.05$ were considered significant.

\section{Results}

\section{Demographic, clinical, and laboratory characteristics of the 59 RA patients}

The patient's characteristics at baseline are summarized in Table 1. The median (IQR) age of the patients was 72 (65-77) years, and the median (IQR) of the RA disease duration was 54 (14-186) months. The median (IQR) of the DAS28-CRP and SDAI were 4.20 (3.39-5.01) and 20.7 (13.7-30.7), respectively. The median (IQR) of the total GS and PD scores were 14 (7-22) and 7 (4-15), respectively. Methotrexate (MTX, median dose: $8 \mathrm{mg}$ weekly) and low-dose oral glucocorticoids (median dose: $5 \mathrm{mg}$ daily) were concomitant in $26(44.1 \%)$ and $33(55.9 \%)$ patients, respectively. Twenty-two (37.3\%) patients had a history of previous use of bDMARDs. 
Table 1

Demographic, clinical and laboratory characteristics of the 59 patients with RA

\section{Age, yrs}

Male, $\mathrm{n}$

Disease duration, months

csDMARDs use, $\mathrm{n}$

MTX use, $\mathrm{n}$

Corticosteroid use, $n$

Previous use of bDMARDs

Oral bisphosphonate use

Positive RF, $n$

Positive ACPA, $n$

Tender joint counts (28), $\mathrm{n}$

Swollen joint counts (28), $\mathrm{n}$

PtGA, mm

EGA, mm

$\mathrm{CRP}, \mathrm{mg} / \mathrm{dl}$

MMP-3, ng/ml

DAS28-CRP

SDAI

Total GS score

Total PD score

The data are median (interquartile range, $Q_{1-4}-Q_{3 / 4}$ ) or number (percentage). ACPA: anti-cyclic citrullinated peptide antibody, bDMARDs: biologic disease-modifying antirheumatic drugs, CRP: Creactive protein, DAS: Disease Activity Score, csDMARDs: conventional synthetic disease-modifying antirheumatic drugs, EGA: evaluator's global assessment, ESR: erythrocyte sedimentation rate, GS: gray-scale, MTX: methotrexate, PD: power Doppler, PtGA: patient's global assessment, RA: rheumatoid arthritis, RF: rheumatoid factor, SDAl: Simplified Disease Activity Index.

\section{Improvement of clinical and MSUS activities over 6 months}

Overall, the patients' DAS28-CRP (Fig. 1A) and SDAI (Fig. 1B) values were significantly improved at 3 and 6 months compared to the baseline ( $p<0.001$, respectively). The median (IQR) of the total GS scores decreased from $14(7-22)$ at baseline to $9(5-18)$ at 3 months $(p<0.05)$ and $9(4-17)$ at 6 months $(p<$ 
0.05) (Fig. 1C). In addition, the median (IQR) of the PD scores decreased from 7 (4-15) at baseline to 4 $(1-11)$ at 3 months $(p<0.05)$ and $2(0-7)$ at 6 months $(p<0.001)($ Fig. $1 D)$.

\section{The serum concentrations of the bone biomarkers}

The results of our comparison of the serum concentrations of the five bone biomarkers between the healthy volunteers and the RA patients and the changes of the biomarkers over the 6-month period after the introduction of abatacept in the RA patients are shown in Supplementary Table S1. Serum OPN was significantly higher $(p<0.0001)$ and serum OC tended to be lower $(p=0.099)$ in the RA patients compared to the healthy volunteers. The other serum bone biomarkers were not significantly different between the RA and healthy groups. Serum OPG was significantly elevated at 6 months after the introduction of abatacept ( $p=0.016$, Suppl. Fig. S1), but the other serum bone biomarkers did not change after treatment. The oral corticosteroids and bisphosphonates did not affect the serum concentrations of bone biomarkers or their changes.

\section{The correlations between the bone biomarkers and disease activity}

Table 2 shows the correlations between the serum levels of the bone biomarkers and the disease activity at baseline and the changes ( $\Delta$ value) over the 6 months of abatacept treatment. At baseline, the serum level of Dkk-1 was positively correlated with the CRP level ( $r s=0.276, p<0.05)$, and that of OC tended to be negatively correlated with the CRP level $(r s=-0.254, p<0.1)$. The serum level of OPG was positively correlated with the SDAl value $(r s=0.264, p<0.05)$ and total GS score $(r s=0.276, p<0.05)$, and it tended to be positively correlated with the total PD score $(r s=0.232, p<0.1)$. 
Table 2

Correlations between serum bone biomarkers and disease activity

\begin{tabular}{|c|c|c|c|c|c|}
\hline \multicolumn{6}{|l|}{ Baseline } \\
\hline & Dkk-1 & SOST & $\mathrm{OC}$ & OPG & OPN \\
\hline CRP & $0.276^{\star \star}$ & -0.137 & $-0.254^{\star}$ & 0.057 & 0.173 \\
\hline MMP-3 & 0.116 & 0.064 & -0.177 & 0.199 & $0.220 *$ \\
\hline DAS28-CRP & 0.078 & -0.088 & -0.004 & 0.178 & 0.119 \\
\hline SDAl & 0.054 & -0.049 & 0.015 & $0.264^{\star \star}$ & 0.146 \\
\hline Total GS score & 0.057 & 0.006 & 0.056 & $0.276^{\star \star}$ & 0.154 \\
\hline Total PD score & 0.027 & -0.107 & -0.042 & $0.232^{\star}$ & 0.096 \\
\hline \multicolumn{6}{|c|}{$\Delta$ value (change over 6 months) } \\
\hline & $\Delta \mathrm{Dkk}-1$ & $\triangle \mathrm{SOST}$ & $\triangle \mathrm{OC}$ & $\triangle \mathrm{OPG}$ & $\triangle O P N$ \\
\hline$\triangle \mathrm{CRP}$ & 0.240 & $-0.264 \star \star$ & -0.161 & $-0.263^{\star \star}$ & 0.057 \\
\hline$\triangle \mathrm{MMP}-3$ & $0.237^{\star}$ & -0.098 & -0.041 & -0.034 & 0.113 \\
\hline$\triangle \mathrm{DAS} 28-\mathrm{CRP}$ & -0.027 & $-0.260 * \star$ & -0.177 & $-0.309 * \star$ & -0.122 \\
\hline$\triangle \mathrm{SDAl}$ & 0.018 & -0.191 & -0.167 & $-0.256^{\star}$ & -0.151 \\
\hline$\Delta$ Total GS score & -0.144 & $-0.223^{\star}$ & -0.030 & $-0.228 *$ & -0.080 \\
\hline$\Delta$ Total PD score & -0.194 & $-0.314^{\star \star}$ & -0.167 & $-0.342^{\star \star \star}$ & -0.127 \\
\hline
\end{tabular}

The $\triangle$ SOST was negatively correlated with the $\triangle \mathrm{CRP}(\mathrm{rs}=-0.264, \mathrm{p}<0.05)$, the $\triangle \mathrm{DAS} 28-\mathrm{CRP}(\mathrm{rs}=-$ $0.260, p<0.05)$, and the $\Delta$ total PD score $(r s=-0.314, p<0.05)$, and it tended to be negatively correlated with the $\Delta$ total GS score $(r s=-0.223, p<0.1)$. The $\triangle O P G$ was negatively correlated with the $\triangle \mathrm{CRP}(\mathrm{rs}=-$ $0.263, p<0.05)$, the $\triangle D A S 28-C R P(r s=-0.309, p<0.05)$, and the $\Delta$ total PD score ( $r s=-0.342, p<0.01)$, and it tended to be negatively correlated with the $\triangle$ SDAI $(r s=-0.256, p<0.1)$ and the $\Delta$ total GS score (rs $=-0.228, p<0.1)$.

\section{The association between the ultrasonographic response and the bone biomarkers}

Because the $\triangle$ SOST and $\triangle \mathrm{OPG}$ were negatively correlated with the $\triangle$ total PD score as described above, we compared these two parameters between the PD responders and non-PD responders. Both the $\triangle S O S T$ and $\triangle O P G$ were significantly greater in the PD responders compared to the non-PD responders $(\mathrm{p}=$ 0.0041 and 0.0073 , respectively, Fig. 2). 
The results of our comparison of the patient characteristics at baseline between the PD responders and non-PD responders at 6 months are provided in Table 3. The patient age and disease duration tended to be higher in the PD responders compared to the non-PD responders. RF positivity $(p=0.030)$, the SDAI ( $p$ $=0.0081)$, the total GS score $(p=0.0005)$, and the total PD score $(p<0.0001)$ were significantly higher in the PD responders compared to the non-PD responders. The serum level of Dkk-1 was significantly lower in the PD responders compared to the non-PD responders $(p=0.026)$. The serum level of SOST tended to be significantly lower in the PD responders compared to the non-PD responders $(p=0.058)$.

Table 3

Comparison of patient characteristics between the PD responders and non-PD responders

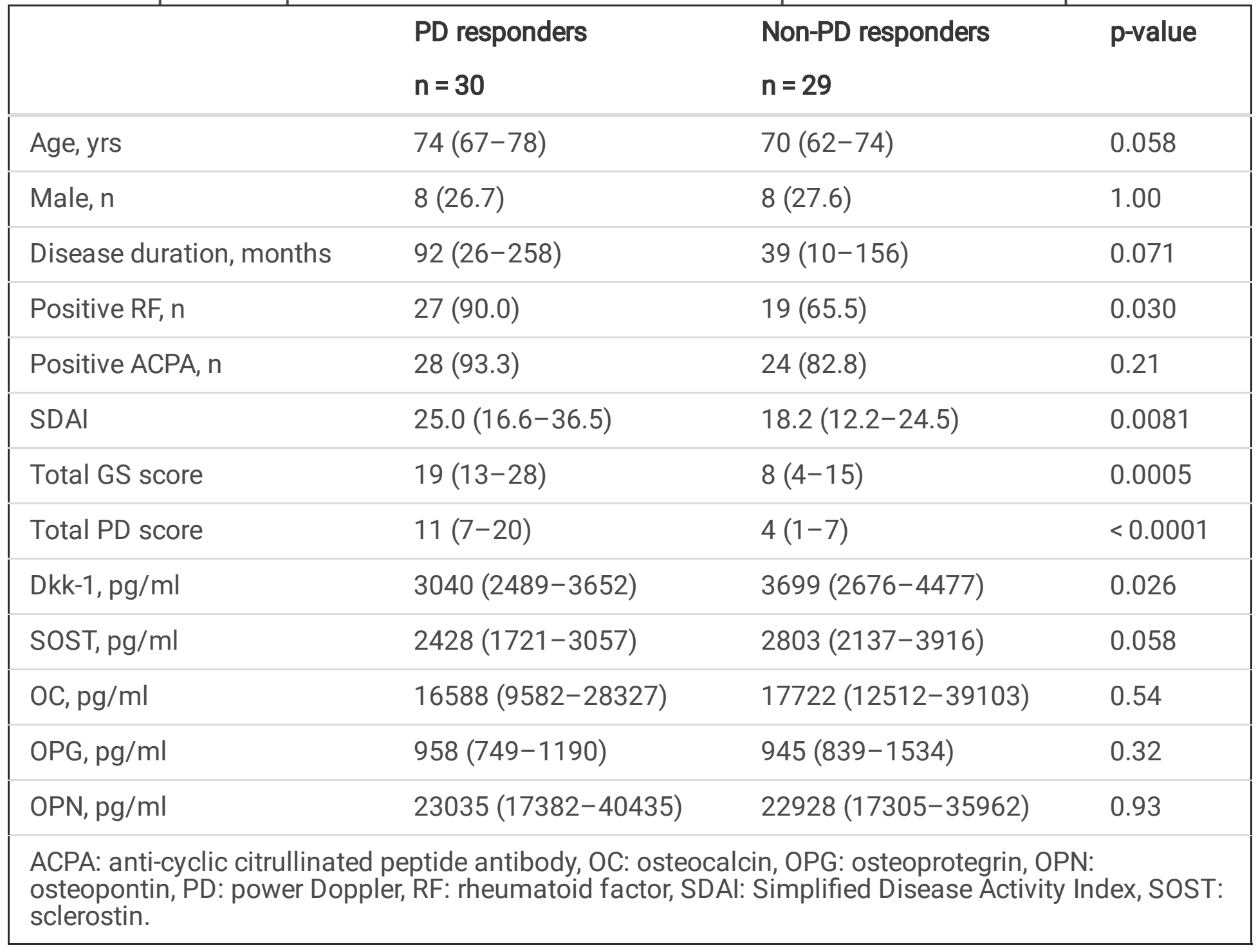

Based on the results of the univariate analysis, we entered six baseline variables (age, disease duration, RF positivity, total PD score, serum Dkk-1 level, and serum SOST level) into the multivariate regressions (Table 4). The serum level of Dkk-1 at baseline (odds ratio [OR] 0.497, 95\% confidence interval [Cl] 0.225$0.909, p=0.043$ ) was revealed as the only independent predictor of PD responder status at 6 months. 
Table 4

Baseline predictors of PD responder at 6 months by multivariate logistic regression analysis

\begin{tabular}{|lllll|}
\hline & Comparison & OR & $95 \% \mathrm{Cl}$ & p-value \\
\hline Serum Dkk-1 & $1 \mathrm{ng} / \mathrm{ml}$ increase & 0.497 & $0.225-0.909$ & 0.043 \\
\hline Serum SOST & $1 \mathrm{ng} / \mathrm{ml}$ increase & 0.562 & $0.286-1.001$ & 0.065 \\
\hline Total PD score & 1 increase & 1.145 & $0.989-1.372$ & 0.098 \\
\hline RF & positive & 3.751 & $0.684-29.05$ & 0.154 \\
\hline Disease duration & 1 year increase & 1.004 & $0.996-1.011$ & 0.343 \\
\hline Age & 1 year increase & 1.009 & $0.934-1.092$ & 0.811 \\
\hline PD: power Doppler, RF: rheumatoid factor, SOST: sclerostin. & \\
\hline
\end{tabular}

We compared the changes in the SDAl and the total PD score between the patients with a low Dkk-1 value (i.e., $<$ the median Dkk-1 value at baseline) and the patients with a high Dkk-1 value ( $\geq$ the median Dkk1 at baseline) (Suppl. Fig. S2) and between the patients with a low SOST value (<the median SOST at baseline) and those with a high SOST value ( $\geq$ the median of SOST at baseline) (Suppl. Fig. S3). The SDAI score improved in the patients with a low Dkk-1 as well as the patients with a high Dkk-1 and in the patients with a low SOST as well as the patients with a high SOST. However, the improvement in the total PD score was better in the patients with a low Dkk-1 compared to those with a high Dkk-1.

\section{Discussion}

We evaluated the association between the serum concentrations of five bone biomarkers and the therapeutic response to abatacept in RA patients, using our multicenter prospective ultrasound cohort study. In the present study, since abatacept significantly improved the patients' clinical disease activity as well as their MSUS score over the 6-month treatment, bone destruction was expected to be prevented in this population.

First, we observed that both Dkk-1 and SOST (which are inhibitors of the Wnt signaling pathway) were associated with the therapeutic response, especially the ultrasonographic response evaluated by the total PD score. Low serum levels of Dkk-1 at baseline was an independent predictor of the PD responder status at 6 months. Low serum levels of SOST at baseline tended to be associated with the PD responder status at 6 months. Decreased serum levels of SOST were significantly correlated with the improvement of disease activity after treatment with abatacept. The Wnt signaling pathway plays a key role in several biological processes such as cellular proliferation and tissue regeneration, and its dysregulation is involved in the pathogenesis of many autoimmune diseases [14]. In RA, Wnt signaling is implicated in systemic and localized bone loss. This process involves proinflammatory cytokines produced by the synovial membrane, which may increase bone resorption but also stimulate soluble antagonists of the 
canonical Wnt/ $\beta$-catenin signaling pathway (including DKK-1 and sclerostin) and subsequently inhibit osteoblast proliferation, maturation, and progenitor differentiation [14, 31, 32].

In particular, the pro-inflammatory cytokines TNF and IL-1 induce Dkk-1 and SOST; IL-17 down-regulates the Wnt/ $\beta$-catenin pathway indirectly, enhancing the production of TNF and IL- 1 ; and IL-6 induces the differentiation of $B$ cells into plasma cells which express Dkk-1 [14, 33, 34]. Moreover, Dkk-1 induces SOST [14]. It has been demonstrated that Dkk-1 promotes synovial angiogenesis, a critical process in the pathogenesis of RA [32]: vascular proliferation occurs during pannus formation in the affected joints [35, 36], during which the synovium becomes locally invasive at the interface with cartilage and bone. The serum level of Dkk-1 was shown to be higher in patients with RA than in controls and to correlate with bone erosions and inflammation [32]. Increased serum levels of Dkk-1 and SOST may therefore indicate a poor prognosis and resistance to treatment in patients with RA. In the present patient series, the serum levels of Dkk-1 and SOST at baseline were closely associated with the responsiveness of PD activity, which reflects synovial angiogenesis [35, 37] and predicts joint destruction [38].

Regarding the effect of bDMARD therapy on the Wnt signaling in RA, most of the relevant studies were conducted with TNFa inhibitors [32,39] or an IL-6 receptor (IL-6R) inhibitor, tocilizumab $[18,19]$, and they showed a decrease in the serum level of Dkk-1 in RA patients undergoing these bDMARD treatments. A reduction in the serum level of Dkk-1 may result from the inhibition of the TNFa- and IL-6-dependent induction of Dkk-1 by TNFa inhibitors or anti-IL- 6 monoclonal antibody. On the other hand, abatacept did not reduce the serum level of Dkk-1 in the present RA patients, perhaps because it does not directly inhibit TNFa and IL-6. There is no report regarding the effect of abatacept, except the present study, on the Wnt signaling in RA patients [14].

In healthy mice, abatacept promoted bone formation by inducing the Wnt ligand Wnt-10b in a T celldependent manner [40], but paradoxically it increased the expression of SOST [41]. Bone formation has been suggested to be moderated by a direct negative feedback loop involving a putative CTLA-4 Ig association with CD80/CD86 on the osteoblasts, causing the production of sclerostin $[14,41]$. In the present patient series, an increased serum level of SOST was significantly correlated with the improvement of disease activity after the introduction of abatacept.

We also observed that the serum level of OPG was significantly elevated after the introduction of abatacept, and this elevation was significantly correlated with the improvement of disease activity. The RANK-RANKL system is the major driver of bone destruction in inflammatory arthritis $[12,14,15]$. This system is promoted by proinflammatory cytokines such as TNFa and IL-6 [12, 14, 15]. OPG, a decoy receptor of RANKL, influences bone erosions in RA [15, 42]. A low OPG/RANKL ratio has thus been associated with increased radiographic damage in RA [15,43]. Wnt signaling is involved in osteoclastogenesis regulation; the canonical Wnt/ $\beta$-catenin signaling pathway leads to an up-regulation of OPG and a down-regulation of RANKL $[14,33]$. TNF inhibitors $[14,44]$ or IL-6R inhibitor $[14,18,19]$ increased both the expression of OPG and the OPG/RANKL ratio, possibly due to a promotion of Wnt signaling following a decrease in DKK-1. Similarly, abatacept may elevate the serum level of OPG 
because it promotes Wnt signaling as described above. In addition, the serum levels of OPN among our patients were higher and those of $\mathrm{OC}$ tended to be lower compared to the healthy volunteers as in previous reports $[21,23]$. However, we did not observe any effect of abatacept treatment on these bone biomarkers.

Some limitations of our study should be mentioned. The limited sample size $(n=59)$ does not allow for subanalyses of differences due to the patients' heterogeneous characteristics. However, our results are valuable as a part of a multicenter collaborative study that prospectively and closely evaluates disease activity using MSUS. Second, we could not evaluate structural changes in joints. In the cohort study, we evaluated the patients' X-ray images at baseline, 6, 12, 18, and 24 months. We will explore whether bone biomarkers are associated with radiographic progression in RA patients treated with abatacept.

\section{Conclusions}

This is the first study to evaluate the effects of abatacept treatment on bone biomarkers in RA patients and to explore whether bone biomarkers are associated with the patients' therapeutic response. Abatacept may prevent bone destruction through the promotion of the Wnt signaling pathway. In addition, the measurements of the serum levels of bone biomarkers may be useful for predicting the ultrasonographic response to abatacept.

\section{Abbreviations}

ACPA: anti-cyclic citrullinated peptide antibody, ACR: American College of Rheumatology, bDMARD: biologic disease-modifying antirheumatic drug, CRP: C-reactive protein, CTLA-4: cytotoxic T lymphocyteassociated antigen-4, DAS: Disease Activity Score, Dkk-1: Dickkopf-1, DMARD: disease-modifying antirheumatic drug, EULAR: European League Against Rheumatism, GS: grayscale, IQR: interquartile range, JCR: Japan College of Rheumatology, MCP: metacarpophalangeal, MSUS: musculoskeletal ultrasound, OC: osteocalcin, OPG: osteoprotegerin, OPN: osteopontin, PD: power Doppler, PIP: proximal interphalangeal, RA: rheumatoid arthritis, SDAl: Simple Disease Activity Index, SOST: sclerostin, T2T: treat-to-target, tsDMARD: targeted synthetic DMARD

\section{Declarations}

\section{Ethical Approval and Consent to participate}

Ethical approval for the study was received from the Institutional Review Board (reference number; 13102866)) of Nagasaki University Hospital. All patients provided written informed consent.

\section{Consent for publication}

Not applicable. 
Availability of supporting data

The collected data processed in this study are stored at the Departments of Immunology and Rheumatology, Nagasaki University Graduate School of Biomedical Sciences, Nagasaki, Japan

\section{Conflicts of Interest}

AK received research funding from Ono Pharmaceutical Co., Sanofi K.K., Asahi Kasei Pharma Corp., Taisho Toyama Pharmaceutical Co., Teijin Pharma Co., Sumitomo Dainippon Pharma Co., Ltd., Chugai Pharmaceutical Co., MSD Co., Kowa Pharmaceutical Co. Ltd., Taisho Toyama Pharmaceutical Co., Pfizer Japan Inc., Kyowa Hakko Kirin Co., Boehringer Ingelheim Japan, Abbvie GK, Astellas Pharma Inc., Eli Lilly Japan, Daiichi Sankyo Co., Takeda Pharmaceutical Co., Kissei Pharmaceutical Co., Mitsubishi Tanabe Pharma Co., Otsuka Pharmaceutical Co., Cosmic Corporation Co. Ltd., Nihon Medi-Physics Co., Ltd., Daiichi Sankyo Co., Bristol-Myers Squibb, Janssen Pharmaceutical K.K., Mochida Pharmaceutical Co., Ltd., AstraZeneca Co., YL Biologics Co., Alexion GK, and Eisai Co. The other authors declare that they have no competing interests.

\section{Funding statement}

This work was supported by Bristol-Myers Squibb and Ono Pharmaceutical Co.

\section{Authors' contributions}

SK and AK made substantial contributions to acquisition of data, statistical analysis, interpretation of data as well as drafting and revision of the manuscript. YE, AN, MO, ST, AT, TS, RS, TK, NI, KI, HN, TA, YU, $T Y, N E, N M, A O, K F, H H, S N$ and $Y T$ carried out acquisition of data, and helped to draft the manuscript. MT, TO and TM contributed to conception and design of the study as well as drafting of the manuscript. All authors read and approved the final manuscript.

\section{Acknowledgements}

We thank all of the members of Kyushu Multicenter Rheumatoid Arthritis Ultrasound Prospective Observational Cohort Study Group for their participation, including Nagasaki University Graduate School of Biomedical Sciences (Shin-ya Kawashiri, Ayako Nishino, Yushiro Endo, Atsushi Kawakami), Sasebo Chuo Hospital (Toshiyuki Aramaki, Yukitaka Ueki), Yoshitama Clinic for Rheumatic Diseases (Tamami Yoshitama), Eiraku Clinic for Rheumatic Diseases (Nobutaka Eiraku), Nagasaki Medical Hospital of Rheumatology Naoki Matsuoka (Naoki Matsuoka), Japanese Red Cross Nagasaki Genbaku Hospital (Akitomo Okada), Japan Community Health care Organization Isahaya General Hospital (Keita Fujikawa), Miyazaki University Hospital (Hiroaki Hamada), Aso lizuka Hospital (Shuji Nagano), Kyushu University Hospital (Yojiro Arinobu), and Saga University Hospital (Syuichi Koarada, Yoshifumi Tada).

\section{References}


1. Scott DL, Wolfe F, Huizinga TW. Rheumatoid arthritis. Lancet. 2010;376:1094-108.

2. Smolen JS, Breedveld FC, Burmester GR, Bykerk V, Dougados M, Emery P, et al. Treating rheumatoid arthritis to target: 2014 update of the recommendations of an international task force. Ann Rheum Dis. $2016 ; 75: 3-15$.

3. Moreland LW, Alten R, Van den Bosch F, Appelboom T, Leon M, Emery P, et al. Costimulatory blockade in patients with rheumatoid arthritis: A pilot, dose-finding, double-blind, placebo-controlled clinical trial evaluating CTLA-4Ig and LEA29Y eighty-five days after the first infusion. Arthritis Rheum. 2002;46:1470-9.

4. Emery P, Burmester GR, Bykerk VP, Combe BG, Furst DE, Barré E, et al. Evaluating drug-free remission with abatacept in early rheumatoid arthritis: Results from the phase $3 \mathrm{~b}$, multicentre, randomised, active-controlled AVERT study of $24+\square$ months, with a 12-month, double-blind treatment period. Ann Rheum Dis. 2015;74:19-26.

5. Schiff M, Weinblatt ME, Valente R, van der Heijde D, Citera G, Elegbe A, et al. Head-to-head comparison of subcutaneous abatacept versus adalimumab for rheumatoid arthritis: Two-year efficacy and safety findings from AMPLE trial. Ann Rheum Dis. 2014;73:86-94.

6. Kremer JM, Russell AS, Emery P, Abud-Mendoza C, Szechinski J, Westhovens R, et al. Long-term safety, efficacy and inhibition of radiographic progression with abatacept treatment in patients with rheumatoid arthritis and an inadequate response to methotrexate: 3-year results from the AIM trial. Ann Rheum Dis. 2011;70:1826-30.

7. Bathon J, Robles M, Ximenes AC, Nayiager S, Wollenhaupt J, Durez P, et al. Sustained disease remission and inhibition of radiographic progression in methotrexate-naive patients with rheumatoid arthritis and poor prognostic factors treated with abatacept: 2-year outcomes. Ann Rheum Dis. 2011;70:1949-56.

8. Matsubara T, Inoue H, Nakajima T, Tanimura K, Sagawa A, Sato Y, et al. Abatacept in combination with methotrexate in Japanese biologic-naive patients with active rheumatoid arthritis: A randomised placebo-controlled phase IV study. RMD Open. 2018;4:e000813.

9. 10.1136/annrheumdis-2019-216655

Smolen JS, Landewé RBM, Bijlsma JWJ, Burmester GR, Dougados M, Kerschbaumer A, et al. EULAR recommendations for the management of rheumatoid arthritis with synthetic and biological diseasemodifying antirheumatic drugs: 2019 update. Ann Rheum Dis 2020. In press. doi:

10.1136/annrheumdis-2019-216655.

10. Axmann R, Herman S, Zaiss M, Franz S, Polzer K, Zwerina J, et al. CTLA-4 directly inhibits osteoclast formation. Ann Rheum Dis. 2008;67:1603-9.

11. Bozec A, Zaiss MM, Kagwiria R, Voll R, Rauh M, Chen Z, et al. T cell costimulation molecules CD80/86 inhibit osteoclast differentiation by inducing the IDO/tryptophan pathway. Sci TransI Med. 2014;6:235ra60.

12. Fardellone P, Séjourné A, Paccou J, Goëb V. Bone remodelling markers in rheumatoid arthritis. Mediators Inflamm. 2014;2014:484280. 
13. Komatsu $\mathrm{N}$, Takayanagi $\mathrm{H}$. Immune-bone interplay in the structural damage in rheumatoid arthritis. Clin Exp Immunol. 2018;194:1-8.

14. Cici D, Corrado A, Rotondo C, Cantatore FP. Wnt signaling and biological therapy in rheumatoid arthritis and spondyloarthritis. Int J Mol Sci. 2019;20:E5552.

15. Szentpétery Á, Horváth Á, Gulyás K, Pethö Z, Bhattoa HP, Szántó S, et al. Effects of targeted therapies on the bone in arthritides. Autoimmun Rev. 2017;16:313-20.

16. Vis M, Havaardsholm EA, Haugeberg G, Uhlig T, Voskuyl AE, van de Stadt RJ, et al. Evaluation of bone mineral density, bone metabolism, osteoprotegerin and receptor activator of the NFkappaB ligand serum levels during treatment with infliximab in patients with rheumatoid arthritis. Ann Rheum Dis. 2006;65:1495-9.

17. Chopin F, Garnero P, le Henanff A, Debiais F, Daragon A, Roux C, et al. Long-term effects of infliximab on bone and cartilage turnover markers in patients with rheumatoid arthritis. Ann Rheum Dis. 2008;67:353-7.

18. Terpos E, Fragiadaki K, Konsta M, Bratengeier C, Papatheodorou A, Sfikakis PP. Early effects of IL-6 receptor inhibition on bone homeostasis: A pilot study in women with rheumatoid arthritis. Clin Exp Rheumatol. 2011;29:921-5.

19. Briot K, Rouanet S, Schaeverbeke T, Etchepare F, Gaudin P, Perdriger A, et al. The effect of tocilizumab on bone mineral density, serum levels of Dickkopf-1 and bone remodeling markers in patients with rheumatoid arthritis. Joint Bone Spine. 2015;82:109-15.

20. Garnero P, Thompson E, Woodworth T, Smolen JS. Rapid and sustained improvement in bone and cartilage turnover markers with the anti-interleukin- 6 receptor inhibitor tocilizumab plus methotrexate in rheumatoid arthritis patients with an inadequate response to methotrexate: Results from a substudy of the multicenter double-blind, placebo-controlled trial of tocilizumab in inadequate responders to methotrexate alone. Arthritis Rheum. 2010;62:33-43.

21. Matuszewska A, Szechiński J. Evaluation of selected bone metabolism markers in rheumatoid arthritis patients. Adv Clin Exp Med. 2013;22:193-202.

22. Sodek J, Chen J, Nagata T, Kasugai S, Todescan R Jr, Li IW, Kim RH. Regulation of osteopontin expression in osteoblasts. Ann NY Acad Sci. 1995;760:223-41.

23. Si J, Wang C, Zhang D, Wang B, Zhou Y. Osteopontin in bone metabolism and bone diseases. Med Sci Monit. 2020;26:e919159.

24. Iwadate $H$, Kobayashi $H$, Kanno T, Asano T, Saito $R$, et al. Plasma osteopontin is correlated with bone resorption markers in rheumatoid arthritis patients. Int J Rheum Dis. 2014;17:50-6.

25. D'Agostino MA, Boers M, Wakefield RJ, Emery P, Conaghan PG. Is it time to revisit the role of ultrasound in rheumatoid arthritis management? Ann Rheum Dis. 2017;76:7-8.

26. Colebatch AN, Edwards CJ, Østergaard M, van der Heijde D, Balint PV, D'Agostino MA, et al. EULAR recommendations for the use of imaging of the joints in the clinical management of rheumatoid arthritis. Ann Rheum Dis. 2013;72:804-14. 
27. Nishino A, Kawashiri SY, Koga T, Iwamoto N, Ichinose K, Tamai M, et al. Ultrasonographic efficacy of biologic and targeted synthetic disease-modifying antirheumatic drug therapy in rheumatoid arthritis from a multicenter rheumatoid arthritis ultrasound prospective cohort in Japan. Arthritis Care Res (Hoboken). 2018;70:1719-26.

28. Endo Y, Koga T, Kawashiri SY, Morimoto S, Nishino A, Okamoto M, et al. Anti-citrullinated protein antibody titre as a predictor of abatacept treatment persistence in patients with rheumatoid arthritis: a prospective cohort study in Japan. Scand J Rheumatol. 2020;49:13-7.

29. Aletaha D, Neogi T, Silman AJ, Funovits J, Felson DT, Bingham CO 3rd, et al. 2010 Rheumatoid arthritis classification criteria: An American College of Rheumatology/European League Against Rheumatism collaborative initiative. Ann Rheum Dis 2010;69(9):1580-8/ Arthritis Rheum 2010;62(9):2569-2581.

30. D'Agostino MA, Boers M, Wakefield RJ, Berner Hammer H, Vittecoq O, Filippou G, et al. Exploring a new ultrasound score as a clinical predictive tool in patients with rheumatoid arthritis starting abatacept: Results from the APPRAISE study. RMD Open. 2016;2:e000237.

31. Singh A, Gupta MK, Mishra SP. Study of correlation of level of expression of Wnt signaling pathway inhibitors sclerostin and dickkopf-1 with disease activity and severity in rheumatoid arthritis patients. Drug Discov Ther. 2019;13:22-7.

32. Wang SY, Liu YY, Ye H, Guo JP, Li R, Liu X, Li ZG. Circulating Dickkopf-1 is correlated with bone erosion and inflammation in rheumatoid arthritis. J Rheumatol. 2011;38:821-7.

33. Diarra D, Stolina M, Polzer K, Zwerina J, Ominsky MS, Dwyer D, et al. Dickkopf-1 is a master regulator of joint remodeling. Nat Med. 2007;13:156-63.

34. Schett G, Stach C, Zwerina J, Voll R, Manger B. How antirheumatic drugs protect joints from damage in rheumatoid arthritis. Arthritis Rheum. 2008;58:2936-48.

35. Strunk J, Heinemann E, Neeck G, Schmidt KL, Lange U. A new approach to studying angiogenesis in rheumatoid arthritis by means of power Doppler ultrasonography and measurement of serum vascular endothelial growth factor. Rheumatology. 2004;43:1480-3.

36. Paleolog EM. Angiogenesis in rheumatoid arthritis. Arthritis Res. 2002;4:81-90.

37. Kawashiri SY, Kawakami A, Iwamoto N, Fujikawa K, Satoh K, Tamai M, et al. The power Doppler ultrasonography score from 24 synovial sites or 6 simplified synovial sites, including the metacarpophalangeal joints, reflects the clinical disease activity and level of serum biomarkers in patients with rheumatoid arthritis. Rheumatology. 2011;50:962-5.

38. Brown AK, Conaghan PG, Karim Z, Quinn MA, Ikeda K, Peterfy CG, et al. An explanation for the apparent dissociation between clinical remission and continued structural deterioration in rheumatoid arthritis. Arthritis Rheum. 2008;58:2958-67.

39. Fassio A, Adami G, Gatti D, Orsolini G, Giollo A, Idolazzi L, et al. Inhibition of tumor necrosis factoralpha (TNF-alpha) in patients with early rheumatoid arthritis results in acute changes of bone modulators. Int Immunopharmacol. 2019;67:487-9. 
40. Roser-Page S, Vikulina T, Zayzafoon M, Weitzmann MN. CTLA-4lg-induced T cell anergy promotes Wnt-10b production and bone formation in a mouse model. Arthritis Rheumatol. 2014;66:990-9.

41. Roser-Page S, Vikulina T, Weiss D, Habib MM, Beck GR Jr, Pacifici R, et al. CTLA-4lg (abatacept) balances bone anabolic effects of T cells and Wnt-10b with antianabolic effects of osteoblastic sclerostin. Ann N Y Acad Sci. 2018;1415:21-33.

42. Schett G, Redlich K, Smolen JS. The role of osteoprotegerin in arthritis. Arthritis Res Ther. 2003;5:239-45.

43. van Tuyl LH, Voskuyl AE, Boers M, Geusens P, Landewé RB, Dijkmans BA, et al. Baseline RANKL:OPG ratio and markers of bone and cartilage degradation predict annual radiological progression over 11 years in rheumatoid arthritis. Ann Rheum Dis. 2010;69:1623-8.

44. Szentpetery A, McKenna MJ, Murray BF, Ng CT, Brady JJ, Morrin M, et al. Periarticular bone gain at proximal interphalangeal joints and changes in bone turnover markers in response to tumor necrosis factor inhibitors in rheumatoid and psoriatic arthritis. J Rheumatol. 2013;40:653-62.

\section{Supplementary Figure Captions}

Suppl. Fig. S1. Changes in the serum levels of OPG over the 6-month abatacept treatment period. Serum OPG was significantly elevated at 6 months after the introduction of abatacept (Wilcoxon signed ranks test). Horizontal bar, median; boxes, 25th and 75th percentiles; bars, 5th and 95th percentiles. OPG: osteoprotegerin.

Suppl. Fig. S2. Comparison of the changes in the SDAl and total PD score between the patients with a low Dkk-1 $(n=30)$ and those with a high Dkk-1 $(n=29)$. Wilcoxon signed ranks test. Horizontal bar, median; boxes, 25th and 75th percentiles; bars, 5th and 95th percentiles. PD: power Doppler, SDAl: Simple Disease Activity Index.

Suppl. Fig. S3. Comparison of the changes in the SDAl and total PD score between the patients with a low SOST $(n=30)$ and those with a high SOST $(n=29)$. Wilcoxon signed ranks test. Horizontal bar, median; boxes, 25th and 75th percentiles; bars, 5th and 95th percentiles.

\section{Figures}


Figure 1

A.

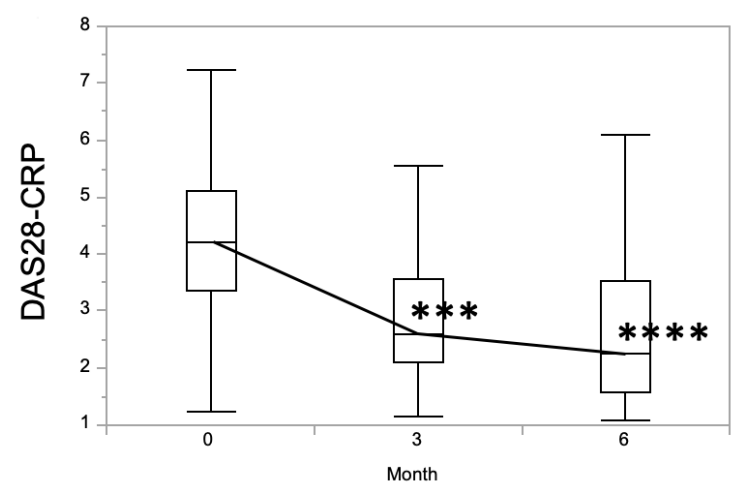

C.

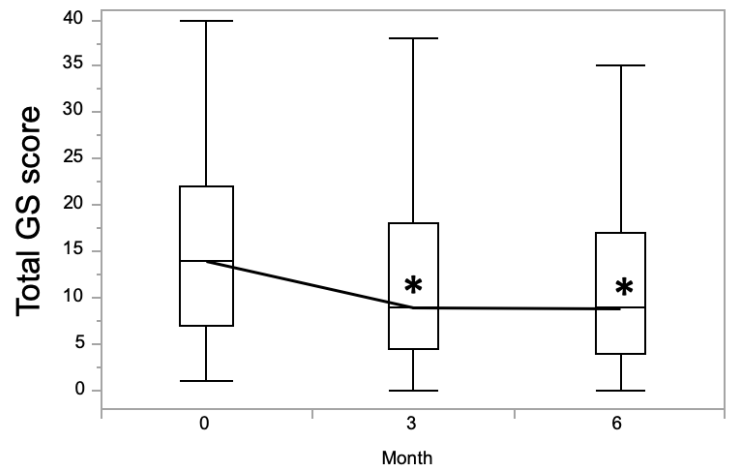

B.

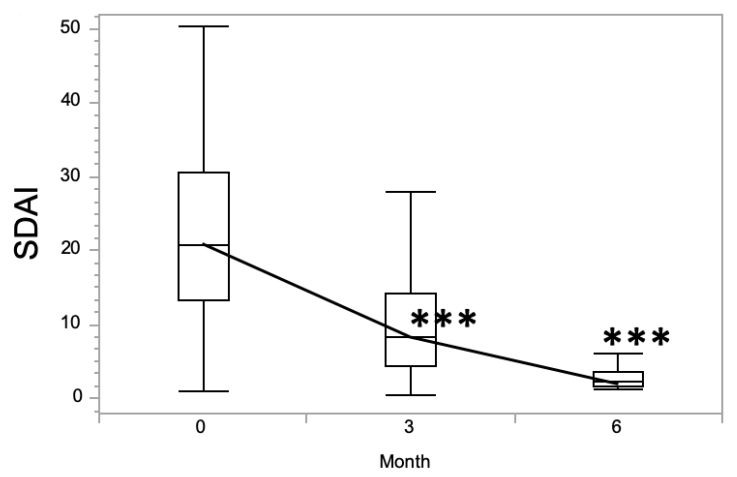

D.

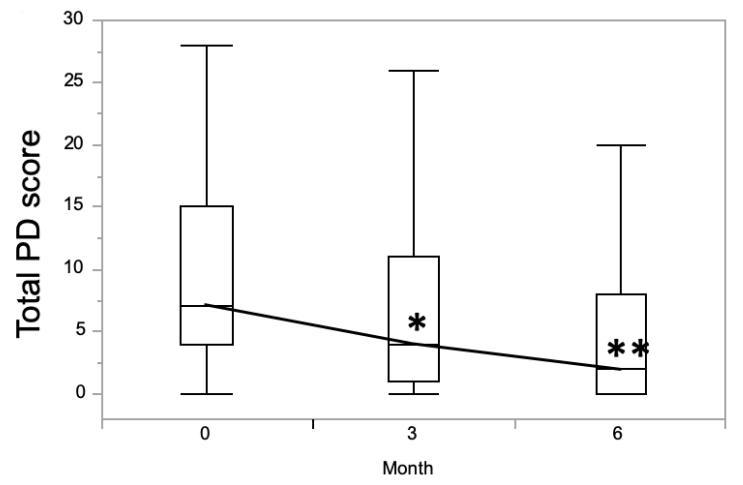

\section{Figure 1}

Changes in the patients' clinical disease activity and MSUS scores over the 6-month abatacept treatment period. The DAS28-CRP (A), SDAI (B), total GS score, and total PD score were significantly improved at 3 and 6 months compared to the baseline. ${ }^{*} p<0.05$, ${ }^{* \star} p<0.001$, ${ }^{\star \star \star} p<0.0001$, Wilcoxon signed ranks test. DAS28-CRP: Disease Activity Score 28-C-reactive protein, GS: grayscale, PD: power Doppler, SDAl: Simple Disease Activity Index. 


\section{Figure 2}

A.

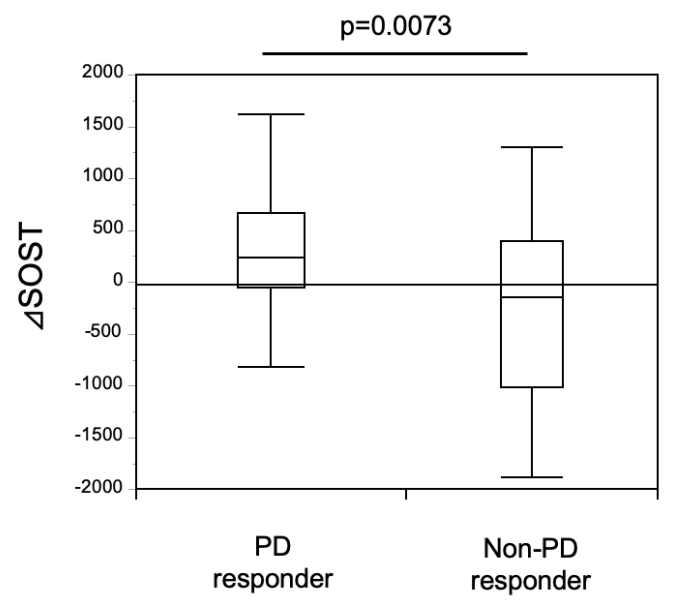

B.

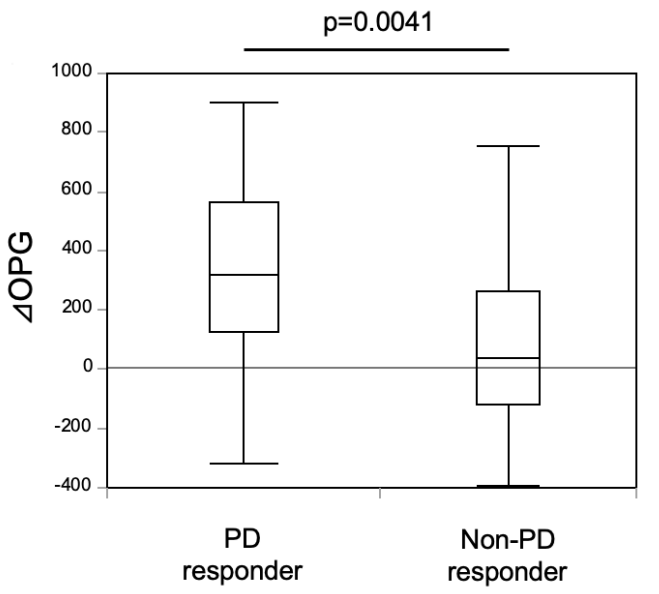

\section{Figure 2}

Comparison of $\triangle S O S T$ and $\triangle O P G$ between the PD responders $(n=30)$ and non-PD responders $(n=29)$. Both the $\triangle S O S T$ and $\triangle O P G$ were significantly greater in the PD responders compared to the non-PD responders. Within-group comparisons were made using Mann-Whitney's U-test. Horizontal bar, median; boxes, 25th and 75th percentiles; bars, 5th and 95th percentiles. OPG: osteoprotegerin, SOST: sclerostin.

\section{Supplementary Files}

This is a list of supplementary files associated with this preprint. Click to download.

- SupplTable.docx

- FigS3.tiff

- FigS2.tiff

- Figs1.tiff 